7 Irvine WJ, Clarke BF, Scarth L, Cullen DR, Duncan LJP. Thyroid and gastric autoimmunity in patients with diabetes mellitus. Lancet 1970; ii:163-8.

${ }^{8}$ Bottazzo GF, Mann JI, Thorogood M, Baum JD, Doniach D. Autoimmunity in juvenile diabetics and their families. $\mathrm{Br}$ Med $\mathcal{F} 1978$; ii: $165-8$.

${ }^{9}$ Bottazzo GF, Florin-Christensen A, Doniach D. Islet cell antibodies in diabetes mellitus with autoimmune polyendocrine deficiencies. Lancet 1974 ;ii:1279-82.

${ }^{10}$ Lendrum R, Walker G, Cudworth AG, et al. Islet cell antibodies in diabetes mellitus. Lancet 1976;ii:1273-6.

11 Cudworth AG, Bottazzo GF, Doniach D. Genetic and immunological factors in type I diabetes mellitus. In: Irvine WJ, ed. Immunology of diabetes. Edinburgh:Teviot Scientific Publications (in press).
12 Bottazzo GF, Cudworth AG, Moul DJ, Doniach D, Festenstein H. Evidence for a primary autoimmune type of diabetes mellitus. $\mathrm{Br} \mathrm{Med} \mathcal{F}$ 1978;ii:1253-5.

13 Joysey VC, Wolf E. HLA-A, $-B$ and $-C$ antigens. Their serology and cross reactions. $\mathrm{Br}$ Med Bull 1978;34:217-22.

14 Irvine WJ, Gray RS, McCallum CJ. Pancreatic islet-cell antibody as a marker for asymptomatic and latent diabetes and pre-diabetes. Lancet 1976;ii :1097-1102.

15 Panayi GS, Wooley P, Batchelor JR. Genetic basis of rheumatoid disease: HLA antigens, disease manifestations, and toxic reactions to drugs. Br Med f 1978;ii:1326-8.

(Accepted 17 October 1979)

\title{
Role of non-medical staff in screening for breast cancer
}

\author{
W D GEORGE, R A SELLWOOD, D ASBURY, G HARTLEY
}

\section{Summary and conclusions}

In a feasibility study of mass population screening for breast cancer by clinical examination and mammography the ability of non-medical staff (nurses and radiographers) to act as primary screeners was compared with that of medical staff (surgeons and radiologists). In 240 women with cancer the rate of detection of the disease by the non-medical staff was comparable to that of the medical staff, although the non-medical staff detected more cancers by mammography alone than did the medical staff. The rate of detection by surgeons and particularly nurses was lower in women without symptoms than in those with symptoms, whereas the incidence of detection by radiologists and radiographers was lower in women aged under 50 than in those over 50. The rate of detection by all groups of staff significantly increased with increases in tumour size.

The results suggest that non-medical staff can act effectively as primary screeners, but that for the detection of cancer in asymptomatic women, particularly those over 50, mammography is probably more effective than clinical examination.

\section{Introduction}

The results of a study by the Health Insurance Plan (HIP) of Greater New York suggested that screening for breast cancer leads to early detection and consequently to a significant reduction in mortality. ${ }^{1}$ Hence the Department of Health and Social Security established three centres to determine the feasibility of providing a national screening service. The results from one of these suggested that it would be possible to provide a safe and acceptable service run by trained non-medical staff. ${ }^{2}$

The success of such a service would depend on the ability of non-medical staff to detect cancer in its early stages in asymptomatic women. This ability may be governed by many factors related to the disease, the population screened, and the methods

Department of Surgery, Royal Liverpool Hospital, Liverpool L7 8XP W D GEORGE, MS, FRCS, senior lecturer

University Hospital of South Manchester, Manchester M20 8LR R A SELLWOOD, CHM, FRCS, professor of surgery

D ASBURY, MB, FFR, consultant radiologist

G HARTLEY, MB, FFR, consultant radiologist of screening used. Within the context of a feasibility study it was possible to examine the effect of several factors on the rate of detection of cancer by both medical and non-medical staff and to consider the effect that these factors may have on large-scale screening programmes.

\section{Subjects and methods}

A breast-screening clinic was established in 1973. The methods chosen for screening were clinical examination and mammography. Nurses and radiographers were recruited and given a basic course of instruction in all aspects of breast disease. Additional instruction in clinical examination was given to the nurses and on interpretation of mammograms to the radiographers. The course lasted six weeks.

\section{POPULATION STUDIED}

Invited women-All women over the age of 40 registered with two group practices were invited to attend the clinic for yearly screening.

Referred women-All patients referred to hospital because of symptoms or signs of breast disease were examined in the same clinic and in the same way as the invited women to provide nonmedical staff with a wide experience of abnormalities of the breast in addition to that gained from "well women."

Self-referred women-Many women attended for screening without invitation or referral.

\section{CLINICAL PROCEDURE}

Each patient was interviewed and examined independently by a nurse and a surgeon, who recorded their findings separately on a standard computer document. In the same way each mammogram was read and reported separately by a radiographer and a radiologist.

\section{PERFORMANCE OF MEDICAL AND NON-MEDICAL STAFF}

The performance of medical and non-medical staff was assessed from their ability to detect significant abnormalities in women with cancer. A significant abnormality was defined as one that automatically led to biopsy. ${ }^{3}$ Histological confirmation was obtained in all cases of cancer.

\section{FACTORS AFFECTING PERFORMANCE}

The effect of the following factors on the rate of detection was studied for each group of staff. 
Symptoms-Most women experience episodes of discomfort and lumpiness of the breast, and a history of these was not regarded as significant. Complaints of a discrete lump in the breast, localised pain, or discharge from the nipple were regarded as significant symptoms for the purposes of this study.

Age of patient-In the HIP study ${ }^{1}$ the benefits of screening were confined to women aged over 50 .

Size of tumour-All tumours were measured and classified according to the TNM system.

\section{STATISTICAL METHODS}

The performance of individual groups of staff for patients subdivided by the presence or absence of symptoms or age was analysed by the method of comparison of proportions ${ }^{4}$ ( $U$ test) corrected for continuity. Performance in relation to tumour size was analysed with a $\chi^{2}$ test for trend. ${ }^{5}$

\section{Results}

DETECTION OF CANCER

A total of 240 women with cancer of the breast was seen and examined by both medical and non-medical staff. Of these women, 213 were among 2325 referred to the clinic, 19 were among 2459 invited to attend (17 at first visit and two at yearly review), and eight were among 1784 who referred themselves. The surgeons detected a significant abnormality in $234(98 \%)$ of the 240 women, and the nurses in $222(93 \%)$. The radiologists detected an abnormality in $185(77 \%)$ of the women and the radiographers in $197(82 \%)$. The joint medical team detected $238(99 \%)$ of the cancers and the joint non-medical team $234(98 \%)$.

\section{FACTORS AFFECTING PERFORMANCE}

\section{Symptoms}

The surgeons recorded the presence of significant symptoms in 193 $(80 \%)$ of the 240 women with cancer, and the nurses in $189(79 \%)$. In women without significant symptoms the rate of detection of a significant abnormality by surgeons and nurses was lower than that in women with symptoms. The surgeons detected a significant abnormality in $191(99 \%)$ of the 193 women with symptoms and in $43(91 \%)$ of the 47 asymptomatic women $(U=2.42 ; p<0.02)$. The nurses detected a significant abnormality in $186(98 \%)$ of the 189 women with symptoms and in $36(71 \%)$ of the 51 asymptomatic women $(U=6.39 ; p<0.001)$. Surgeons were more accurate than nurses in detecting significant abnormalities in asymptomatic women $(91 \%$ and $71 \%$ respectively).
The performance of radiologists and radiographers was not affected by the presence or absence of recorded symptoms. The radiologists detected a significant abnormality in $148(77 \%)$ of the 193 women in whom the surgeons had recorded symptoms and in $37(79 \%)$ of the 47 asymptomatic women. Radiographers detected a significant abnormality in $155(82 \%)$ of the 189 women in whom the nurses had 3 recorded symptoms and in $42(82 \%)$ of the 51 asymptomatic women.

The performance of the joint medical team was not affected $\stackrel{.}{.}$ significantly by the presence or absence of symptoms, but that of the joint non-medical team was significantly less accurate in asymptomatic $\hat{\sigma}$ women. Cancers were detected by the joint medical team in $192(99 \%)$ t of the 193 women in whom the surgeons had recorded symptoms and in $46(98 \%)$ of the 47 asymptomatic women.

A significant abnormality was detected by the joint non-medical team in $187(99 \%)$ of the 189 women in whom the nurses had recorded $\odot$ symptoms, and in $47(92 \%)$ of the 51 asymptomatic women $(\mathrm{U}=2 \cdot 25$; \& $\mathrm{p}<0.05)$. In the 189 symptomatic women, only one $(0.5 \%)$ of the $187 \overrightarrow{.}$ cancers would have been missed without mammography, but in the asymptomatic women $11(23 \%)$ of the 47 cancers would have been missed. This difference was significant $(U=5.98 ; p<0.001)$.

Age

Of the 240 cancers, $53(22 \%)$ were in women under $50,67(28 \%)$ in women aged $50-59,71(30 \%)$ in women aged $60-69$, and $49(20 \%)$ in women aged 70 and over.

The rate of detection by surgeons and nurses was not affected by age, but the rate of detection by radiologists and radiographers was lower in women under 50 than in those over 50 (table I). The rate of $\frac{O}{I}$ detection by radiologists in women under 50 was $62 \%$ and in those $\overrightarrow{0}$ over $5082 \%$. This difference was significant $(U=2.63 ; p<0.01)$. The 6 rate of detection by radiographers in women under 50 was $74 \%$ and in those over $5085 \%$. This trend was not significant.

\section{Size of tumour}

Of the 240 tumours, $26(11 \%)$ were classified as $T_{1}$ by size, 110 $(46 \%)$ as $\mathrm{T}_{2}, 82(34 \%)$ as $\mathrm{T}_{3}$, and $22(9 \%)$ as $\mathrm{T}_{4}$. The rate of detection increased significantly with the size of the tumour for all groups of staff (table II). A similar trend was seen with the rates of detection by the joint medical and joint non-medical teams, but even with the $\stackrel{\circ}{\varnothing}$ smallest tumours $\left(\mathrm{T}_{1}\right)$ their rates of detection were high, being $96 \%$ ○ for the joint medical team and $88 \%$ for the joint non-medical team.

\section{CANCERS IN POPULATION GROUPS STUDIED}

Seventeen cancers were detected in the 2459 women who attended for screening by invitation. A further two cancers were detected in

TABLE I-Age distribution of 240 women with breast cancer detected by medical and non-medical staff. Figures are numbers of cases (percentages in parentheses)

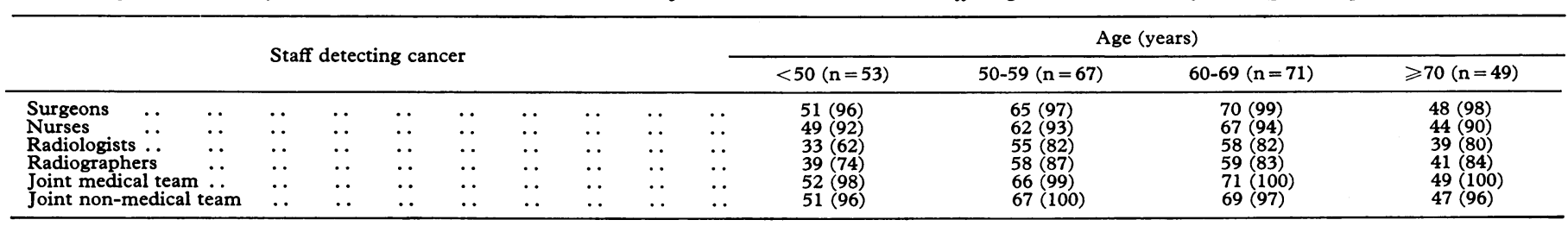

TABLE II-Sizes of tumour (TNM classification) detected by medical and non-medical staff in 240 women with breast cancer. Figures are numbers of cases percentages in parentheses

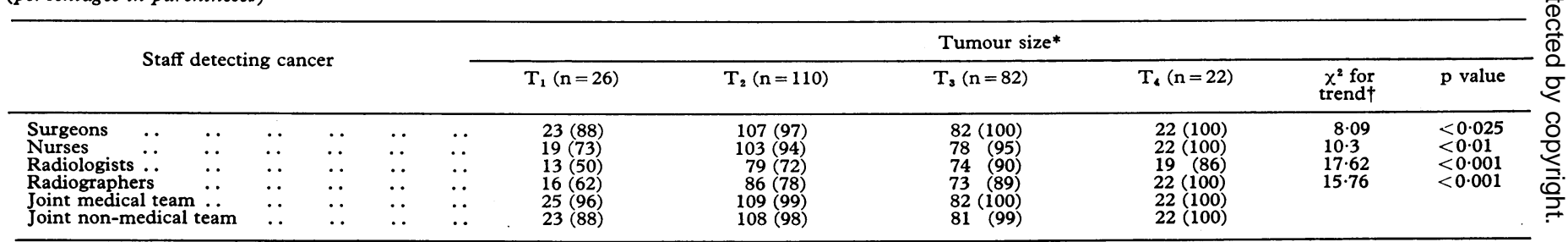

Tumour sizes classified according to TNM system.

$\dagger \chi^{2}$ tests performed with two degrees of freedom. 
1575 of these women who attended for yearly review. Only one of the cancers was detected in a woman under 50 . The accuracy of the surgeons and nurses in these cancers was considerably less than that in the total series. The surgeons detected a significant abnormality in 15 of the 19 women with cancer, the nurses detected 11 , and the radiologists and radiographers both detected 16 . The joint medical team detected all 19 cancers and the joint non-medical team 17 .

Both surgeons and nurses recorded symptoms in nine of the 19 women with cancer, the remaining 10 being asymptomatic. The surgeons detected a significant abnormality in seven of these asymptomatic women, the nurses in two, the radiologists in nine, the radiographers in eight, the joint medical team in all of these women, and the joint non-medical team in nine. Three of the four cancers missed by the surgeons and each of the eight missed by the nurses had not caused symptoms.

Mammography was thus more valuable in the invited women than in those who attended for other reasons. Of the 219 cancers detected by the joint medical team in women who attended other than by invitation, $169(77 \%)$ were detected by both clinical examination and mammography, $50(23 \%)$ by clinical examination alone, and none by mammography alone. Of the 19 cancers detected by the joint medical team in invited women, $12(63 \%)$ were detected by both clinical examination and mammography, three $(16 \%)$ by clinical examination alone, and four $(21 \%)$ by mammography alone. The difference in the rate of detection by mammography between the two groups was significant $(U=5.92 ; p<0.001)$.

Of the 217 cancers detected by the joint non-medical team in patients who attended other than by invitation, $175(81 \%)$ were detected by both clinical examination and mammography, $36(16 \%)$ by clinical examination alone, and $6(3 \%)$ by mammography alone. Of the 17 cancers detected by the joint non-medical team in invited patients, $10(59 \%)$ were found by both clinical examination and mammography, $1(6 \%)$ by clinical examination alone, and $6(35 \%)$ by mammography alone. The difference in the rate of detection by mammography between the two groups was significant $(U=5 \cdot 28$; $\mathrm{p}<0.001)$.

\section{Discussion}

In women with cancer the nurses and radiographers together detected significant abnormalities in the breast as effectively as the medical staff. The nurses were slightly less successful in detection than the surgeons, and the radiographers were slightly more successful in detection than the radiologists. Thus the joint non-medical team detected relatively more cancers by mammography only than did the joint medical team. The performance of the surgeons and nurses was affected by the presence or absence of symptoms, and the performance of the radiologists and radiographers by the age of the women. Small tumours were detected less often than large ones by all groups.

Most women with cancer were referred to the clinic, and many had symptoms which drew attention to an abnormality in the breast. In these women the accuracy of surgeons and nurses was high. In asymptomatic women, who predominate in a population invited for screening, the accuracy of surgeons and nurses was significantly less than in those with symptoms. The performance of the radiographers and radiologists was not affected by symptoms but was affected by age. They were less likely to detect significant abnormalities in women under 50 than in those over 50 . This was probably because of difficulty in detecting small lesions in relatively dense premenopausal breasts. This difficulty has been reported before. ${ }^{6} 7$ The rate of detection of cancer by all groups of staff increased with the size of the tumour.

Although the performance of the various groups of staff was affected by symptoms, age of the patient, and size of the tumour, the incidence of detection of cancer by the joint nonmedical team was similar to that of the joint medical team in each group of women. This suggests that nurses and radiographers can act effectively as primary screeners, but the factors that influence their performance have considerable implications for a screening service.

The lower rate of detection by the nurses in asymptomatic women emphasised the role of mammography in such women.
The purpose of screening is to detect cancers before they produce symptoms, and without mammography the joint nonmedical team would have missed one-fifth of the cancers they detected in women in whom the nurses had not recorded symptoms. Mammography, however, was less effective in women under 50 . This suggests that screening will probably be less effective in women under 50 than in those over 50 and confirms the findings of the HIP study in New York. The lower rate of detection by mammography in younger women may explain why the benefits of screening were confined to women over 50 in the HIP study.

In our series 19 cancers were detected in women invited to attend for screening. Only one of these was detected in women aged 40-49; this was detected by both radiographer and radiologist and missed by both surgeon and nurse. Of the 19 invited women with cancer, nine had symptoms. The nurses detected cancers in all of these nine and the surgeons in eight. The remaining 10 cancers were detected in asymptomatic women and reflect the true aims of a screening service. Of these 10, three were missed by surgeons and eight by nurses. This inaccuracy re-emphasises the role of mammography in screening. Over one-third of all cancers detected by the non-medical team in women invited for screening were detected by mammography alone.

Our results suggest that in screening services run by trained non-medical staff mammography will be more successful than clinical examination in detecting cancer in asymptomatic women. Because of this, and because conventional mammography is less accurate in women under 50 than in those over 50, the validity of including younger women in screening programmes must be doubted. Contrastingly, it is difficult to exclude women aged 40-50 from such programmes because of their high incidence of cancer and high rate of participation. Improvements in imaging techniques may resolve this dilemma.

We thank the DHSS for their generous grant, which made this work possible, and for their helpful advice and support throughout the project. We are grateful to the staff of the regional computer centre for their help; to Dr Blumfield, Dr McShane, and their colleagues in general practice; to the nurses, radiographers, and secretaries in the breast clinic; and to Mrs Patricia Clark for secretarial help.

\section{References}

1 Shapiro S, Strax P, Venet L, Venet W. Changes in 5-year breast cancer mortality in a breast-screening programme. In: Seventh national cancer conference proceedings. Philadelphia: BJ Lippincott, 1973:663-78.

${ }^{2}$ George WD, Gleave EN, England PC, et al. Screening for breast cancer. Br Med F 1976;ii:858-60.

${ }^{3}$ George WD. The feasibility of screening for cancer of the breast. Thesis. London: University of London, 1977.

${ }^{4}$ Armitage P. Statistical methods in medical research. Oxford: Blackwell, 1976:126.

5 Armitage P. Statistical methods in medical research. Oxford: Blackwell, 1976:363.

${ }^{6}$ Friedman AK, Askovitz SI, Berger SM, et al. A co-operative evaluation of mammography in seven teachin 5 hospitals. Radiology 1966 ; 86:886-91.

${ }^{7}$ James WB, Irvine RW. Mammography in the management of breast lesions. Br Med $\mathcal{F} 1969$;ii:655-7.

(Accepted 12 October 1979)

ONE HUNDRED YEARS AGO On the 27th of last month, a distressing occurrence took place in Glasgow, which resulted in the death of a girl aged four years. Unobserved by the parents, she obtained possession of a box of lucifer matches, with which she amused herself for some time. Shortly afterwards, she seemed somewhat indisposed, and, though an examination of the box showed that the child had been sucking the phosphorus on the matches, not much was thought of the occurrence. As the child, however, grew worse, medical advice was obtained; but she gradually sank, and died early on the following morning. (British Medical fournal, 1880.) 